\title{
Pulse oximetry screening: a review of diagnosing critical congenital heart disease in newborns
}

This article was published in the following Dove Press journal:

Medical Devices: Evidence and Research

II July 2016

Number of times this article has been viewed

\author{
Melissa S Engel' \\ Lazaros K Kochilas ${ }^{2}$ \\ 'Division of Neonatology, University \\ of Minnesota, Minneapolis, MN, \\ ${ }^{2}$ Children's Healthcare of Atlanta \\ Emory University, Atlanta, GA, USA
}

\begin{abstract}
Congenital heart disease (CHD) is one of the most common birth defects, with an incidence of nine out of every 1,000 live births. The mortality of infants with CHD has decreased over the past 3 decades, but significant morbidity and mortality continue to occur if not diagnosed shortly after birth. Pulse oximetry was recommended as a screening tool to detect critical CHD in 2011 by the American Academy of Pediatrics and the American Heart Association. Pulse oximetry is a tool to measure oxygen saturation, and based on the presence of hypoxemia, many cardiac lesions are detected. Due to its ease of application to the patient, providing results in a timely manner and without the need for calibrating the sensor probe, pulse oximetry offers many advantages as a screening tool. However, pulse oximetry has also important limitations of which physicians should be aware to be able to assess the significance of the pulse oximetry measurement for a given patient. This review aims to highlight the benefits and shortcomings of pulse oximetry within the context of screening for critical CHD and suggests future avenues to cover existing gaps in current practices.
\end{abstract}

Keywords: congenital heart disease, critical congenital heart disease, pulse oximetry, newborn, newborn screening

\section{Introduction}

Congenital heart disease (CHD) is one of the most common birth defects, with an incidence of nine out of every 1,000 live births. ${ }^{1,2}$ Critical CHD (CCHD) is defined as cardiac lesions that require surgery or cardiac catheterization within the first month (or within the first year by different definitions) of life to prevent death or severe endorgan damage. ${ }^{2}$ Although infant mortality has decreased over the past 3 decades for children with all forms of CHD, many children are still diagnosed too late to avoid significant morbidity or death. ${ }^{3-5}$ Delayed diagnosis of CCHD is unfortunately all too common, with up to $25 \%$ of infants with these defects being missed in newborns when identification is based on clinical symptoms or signs of heart disease even in settings with routine prenatal sonograms. ${ }^{3,6-8}$ Approximately $40 \%$ of these infants with missed diagnoses at birth present in cardiogenic shock at a medical facility and $5 \%$ are diagnosed at autopsy., ${ }^{4,9}$ Studies in Europe and the US have suggested that newborn screening with pulse oximetry testing prior to discharge from the nursery can decrease the number of missed diagnoses by $\sim 30 \%{ }^{2,10}$ In 2011 , pulse oximetry screening for CCHD was added to the Recommended Uniform Screening Panel by the Health and Human Services Secretary. ${ }^{11}$ In the subsequent years, many states have implemented their own protocols to comply with this recommendation. ${ }^{12-15}$ 
Pulse oximetry has been the mainstay procedure for indirectly detecting hypoxemia in medically ill patients since the 1980s. ${ }^{16,17}$ The screening of CCHD by pulse oximetry involves taking advantage of its ability to detect clinical and more importantly subclinical levels of hypoxemia that should raise suspicion for a CCHD. However, pulse oximetry does not readily offer information about decreased stroke volume, which is another physiologic feature of several CCHDs that could be detectable during the neonatal transition. In this review, we highlight the relevant principles and limitations of pulse oximetry in the context of detecting CCHD.

\section{Pulse oximetry: principles of operation and limitations}

The Beer-Lambert-Bouguer law of physics describes the attenuation of light to the properties of materials through which the light is traveling. ${ }^{16}$ Oxygenated blood absorbs red light at a wavelength of $660 \mathrm{~nm}$, and deoxygenated blood absorbs light in the infrared spectrum at $940 \mathrm{~nm} .{ }^{16}$ Computation of oxygen saturation is achieved with the use of calibration algorithms, based on the amount of signals from nonpulsatile (venous, capillary, bone, and skin) and pulsatile arterial blood flow, in the red and infrared wavelengths mentioned earlier. A microprocessor removes the continuous signal from the nonpulsatile tissues and vessels, which leaves the pulsatile signal from the arteries to be displayed as a plethysmographic wave form on the pulse oximeter monitor. ${ }^{16}$

Safe use of pulse oximetry requires knowledge of its limitations. There are multiple sources of potential artifacts, which are particularly relevant for neonates and can cause false readings. These include motion artifacts, poor perfusion and cold skin at the site of measurement, irregular rhythms, ambient light, phototherapy or electromagnetic interference, skin pigmentation and jaundice, inappropriate probe positioning (penumbra effect), venous pulsation, intravenous dyes, and presence of abnormal hemoglobin molecules. ${ }^{16-18}$

Pulse oximeters recommended for screening for CHD should report functional oxygen saturation (referring to the hemoglobin that is capable of transporting oxygen), be motion "tolerant", be validated for low perfusion states, and have a $2 \%$ root-mean-square accuracy. ${ }^{11}$ Of note, a pulse oximeter's performance is optimized for oxygen saturations $\left(\mathrm{SpO}_{2}\right)$ in the range of $80 \%-100 \%$. The development of the modern pulse oximeter is based on healthy, fit adult individuals who were exposed to different degrees of subambient oxygen with their $\mathrm{SpO}_{2}$ being kept between $80 \%$ and $100 \%$. Therefore, any $\mathrm{SpO}_{2}<80 \%$ is extrapolated by a computer program. ${ }^{19,20}$ Most new pulse oximeters are able to detect motion and label it as artifact or perform calculations quickly in a way that renders them motion tolerant. The $\mathrm{SpO}_{2}$ value is not a continuous assessment. The pulse oximeter uses an algorithm to average readings over a period of time. For the most accurate measurement, the average is taken over a shorter period of time, which also increases the delay of the reading and decreases the speed of computation by the machine. For a pulse oximetry measurement to be accurate, the peripheral tissue needs an adequate pulse volume and pressure. In situations such as septic shock, where the extremities are cool and have low perfusion, the pulse oximeter may not reliably assess the oxygen saturation. ${ }^{19,20}$

Competing light sources, such as other machinery, fluorescent lighting, and even cell phones, can "overload" the semiconductor sensor. In pediatrics, a phenomenon called the "penumbra effect" occurs. ${ }^{18}$ Pulse oximeters may overor under-read the $\mathrm{SpO}_{2}$ in infants and children because of the small size of their fingers, or the other areas where the pulse oximeter probe is placed, and the different light paths for each wavelength through the peripheral tissue. Specific probes for infants and children have been created and should be used to prevent these false measurements. Finally, pulse oximetry should not be used in certain circumstances in which the hemoglobin molecule is not completely saturated with oxygen, such as with carbon monoxide poisoning or when methemoglobinemia occurs after administration of certain medications such as antimalarial drugs, nitrates, nitrites, or dyes such as methylene blue. In these instances, co-oximetry is the only reliable method to measure oxygen carried by blood. ${ }^{16,19,20}$

\section{Pulse oximetry in neonates}

Pulse oximetry is used in all aspects of newborn care, including resuscitation of newborns in the delivery room to routine monitoring in the operating room. A study by Levesque et $\mathrm{al}^{21}$ in 2000 described the normal range of oxygen saturations in term newborns in the first days of life. These investigators evaluated normal oximetry values at sea level, from admission to the newborn nursery to discharge. They also evaluated variables such as sex, gestational age at birth, birth weight, mode of delivery, Apgar scores, pre- or postductal site of measurement, and status of the infant at the time of measurement (sleeping, quiet, and crying). Pulse oximetry measurements were taken upon admission to the newborn nursery, at 24 hours of life, and at discharge. The overall oxygenation saturation was 97.2\% $\pm 1.6 \%(95 \%$ CI $97.1 \%-97.2 \%) .{ }^{21}$ They compared the mean values at each time period, which 
showed a slight increase in oxygen saturation over time for the right hand (preductal) and right foot (postductal). Rising from statistical significance were only two variables, postnatal age and activity of the infant. Postnatal age was found to be statistically significant $(+0.17 \%$ per 24 -hour interval from admission to discharge, $P=0.0001$ ) but not clinically relevant. ${ }^{21}$ Infant's activity was statistically significant with values obtained while the infant was crying, fussy, or awake being lower than the values obtained while sleeping. ${ }^{21}$

\section{Pulse oximetry use for CCHD screening}

de Wahl Granelli et al ${ }^{10,22}$ systematically measured the oxygen saturation in 40,000 newborns and compared the values obtained in children with CCHD vs otherwise healthy newborns. In addition, these investigators showed that two of the three infants with CCHD were missed by physical examination alone. ${ }^{22}$ Addition of pulse oximetry screening raised the diagnosis rate to $82 \%$. Several other studies mostly from Europe revealed similar findings. ${ }^{12,23-28}$ These were summarized in a systematic review and meta-analysis of pulse oximetry screening for CCHD in the newborn nursery, which included 13 studies with 229,421 infants. ${ }^{28}$ Sensitivity of pulse oximetry was $76.5 \%$ (95\% CI 67.7-83.5) and specificity was $99.9 \%$ (95\% CI 99.7-99.9) for the detection of $\mathrm{CHD}$, with the average false-positive rate for these infants being $0.14 \%$ (95\% CI $0.16-0.33){ }^{28}$

With the average pulse oximetry value being $97.2 \%$ during the first days of life for all newborns, pulse oximetry is an excellent tool to evaluate subclinical hypoxemia, that occurs during transitioning physiology of certain CHD, such as transposition of the great arteries, truncus arteriosus communis, hypoplastic left heart syndrome, total anomalous pulmonary venous connection, tricuspid atresia, tetralogy of Fallot, and pulmonary atresia. These lesions are usually associated with hypoxemia in the newborn period and can cause significant morbidity and mortality if the diagnosis is delayed. In September 2010, the Secretary's Advisory Committee on Heritable Disorders in Newborns and Children (SACHDNC) considered these seven lesions as primary targets for pulse oximetry screening in the newborn period on the basis of advice from a technical expert panel. ${ }^{2}$ In 2011, the SACHDNC, in collaboration with the American Academy of Pediatrics, the American College of Cardiology Foundation, and the American Heart Association, convened a work group to outline implementation strategies for pulse oximetry screening in newborns for CHD. After reviewing data from existing large studies in Sweden and the UK, ${ }^{22,25}$ the work group proposed a screening protocol based on results of measurements from the right hand (preductal) and either foot (postductal). ${ }^{11}$ According to the SACHDNC protocol, an infant would have a positive (failing) screen if at $\geq 24$ hours of life: 1) A pulse oximeter reading was $<90 \%$ in either the right hand or either foot. 2) Both readings from the right hand and either foot were $<95 \%$ on three measurements each separated by 1 hour. 3) A persistent $>3 \%$ difference in the right hand and either foot measurement on three measurements each separated by 1 hour.

An infant who had $\geq 95 \%$ in either extremity with $\leq 3 \%$ difference in the pre- and postductal oxygen saturation would have a negative screen and no further work-up is needed. Many states that have initiated pulse oximetry screening have adapted this protocol or a variation there of. ${ }^{12,13,15}$ Kochilas et $\mathrm{al}^{14}$ demonstrated that the SACHDNC protocol was the most efficient protocol with the fewest false-positive pulse oximetry screens in the newborn period. Data from various studies suggest that this protocol is adequate to detect clinical and subclinical hypoxemia that is associated with the seven primary target lesions based on their almost universal association with at least mild hypoxemia in the neonatal period, when the fetal circulation transitions to postnatal circulation. The protocol should also be effective, although to a lesser degree, to detect the hypoxemia associated with five additional congenital heart lesions that are considered as secondary targets. ${ }^{6,9}$ These lesions are frequently associated with at least some degree of neonatal hypoxemia and include proximal aortic arch anomalies (such as interrupted aortic arch or aortic atresia), coarctation of the aorta (CoA) with patent ductus arteriosus, Ebstein's anomaly, double outlet right ventricle, and single ventricle lesions. There are additional lesions that are "possibly screenable" in the neonatal period with the same protocol based on their anatomy and potential for intracardiac or ductal-level shunting. These include aortic stenosis with a patent ductus arteriosus, severe pulmonary stenosis, and complete common atrioventricular canal. Finally, there is a category of cardiac lesions that will not have hypoxemia in the newborn period and can be classified as "not screenable" and include left-sided obstructive lesions such as CoA without patent ductus arteriosus and aortic stenosis without a patent ductus arteriosus, Ebstein's anomaly without interatrial shunting from right-to-left, and all other lesions that cause left-to-right shunting and valve anomalies that were not included in the previous categories ${ }^{29}$ (Table 1).

Although not all CHDs cause hypoxemia, these types of lesions can still lead to serious complications if not detected 
Table I Congenital heart disease lesions divided by likelihood of being detected by pulse oximetry

\begin{tabular}{|c|c|c|c|}
\hline Primary targets & Secondary targets & Possibly screenable & Not screenable \\
\hline Hypoplastic left heart syndrome & $\begin{array}{l}\text { Interrupted aortic arch/aortic } \\
\text { atresia }\end{array}$ & Aortic stenosis with PDA & $\begin{array}{l}\text { Coarctation of the aorta } \\
\text { without a PDA }\end{array}$ \\
\hline Pulmonary atresia & Coarctation of the aorta with PDA & Pulmonary stenosis & $\begin{array}{l}\text { Ebstein's anomaly without } \\
\text { right-to-left shunt }\end{array}$ \\
\hline $\begin{array}{l}\text { Total anomalous pulmonary venous } \\
\text { connection }\end{array}$ & Ebstein's anomaly & Complete atrioventricular canal & Aortic stenosis without PD/ \\
\hline Transposition of the great arteries & Double-outlet right ventricle & & $\begin{array}{l}\text { Other left-to-right shunting } \\
\text { lesions }\end{array}$ \\
\hline Tetralogy of Fallot & Single ventricle physiology & & \\
\hline \multicolumn{4}{|l|}{ Tricuspid atresia } \\
\hline Truncus arteriosus communis & & & \\
\hline
\end{tabular}

Notes: Primary and secondary targets are cardiac lesions that will have hypoxemia in the newborn period. Lesions are considered possibly screenable or not screenable because of lesser degree of hypoxemia.

Abbreviation: PDA, patent ductus arteriosus.

early enough to avoid end-organ damage. Among them, the most frequent category is comprised of some form of leftsided obstructive lesions for which additional diagnostic strategies may soon become clinically available. One of the most promising ones is the peripheral perfusion index (PPI), which is based on the analysis of the pulse oximetry signal and is displayed on some newer generation pulse oximeters. More specifically, this technique uses the ratio between the pulsatile and nonpulsatile component of the pulse oximetry signal to detect changes in the relative amount of arterial perfusion in the examined site. ${ }^{29,30}$ As measured, the PPI may be useful in detecting reduction in the arterial circulation at the monitoring site and can be used to detect decreased perfusion in settings of left heart obstructive lesions either globally (ie, aortic stenosis) or regionally (CoA). Important challenges for this technique remain the wide and highly skewed distribution of PPI values in the normal population and sensitivity to environmental factors such as skin temperature. Preliminary work with PPI in neonates has established reference values for newborns with cutoff values of $<0.70$ for possibly impaired peripheral perfusion and $<0.50$ definite hypoperfusion. ${ }^{31}$ However, further studies with children with various cardiac lesions are needed before incorporating PPI in the screening process for CCHD.

Few studies have shown the cost-effectiveness of pulse oximetry screening in the newborn nursery. Two studies published by Peterson et a $\mathrm{l}^{32}$ estimated that routine screening of newborns would identify an additional 1,189 infants with CCHD at their respective birth hospitals that would not have been diagnosed prior to discharge. In addition, they estimated that 20 infant lives would be saved each year by screening and a cost of $\sim \$ 40,385$ per life-year will be gained. ${ }^{32}$ The estimated cost for pulse oximetry screening in the newborn nurseries in New Jersey was $\$ 14.09$ per newborn, with supplies and labor dividing this cost almost equally. ${ }^{33}$ Peterson et $\mathrm{al}^{33}$ extrapolated that the estimated average cost to screen all newborns in the US regardless of which level of nursery they were in was $\$ 13.50$ per newborn.

In the scientific statement from the American Heart Association and the American Academy of Pediatrics, Mahle et al evaluated the statistical analysis of pulse oximetry screening with data from ten different studies. Analysis on the studies with infants who were evaluated after 24 hours of age showed 18 false positives, along with seven false negatives and 51,063 true negatives. With these data, the sensitivity of pulse oximetry was $69.9 \%$ and the specificity was $99.9 \%$, with a negative predictive value of $99.9 \%$ and a positive predictive value of $47 \% .{ }^{2}$ Pulse oximetry is an adequate screening tool, with few false positives and a high negative predictive value.

As far as we know at the time of writing this article, there are no sufficient data available on the burden to the health care system with the increase in infant echocardiograms completed due to failed pulse oximetry screening in the nursery.

\section{Conclusion}

Each type of CHD affects cardiopulmonary circulation differently, and pulse oximetry is a good screening tool to detect lesions that cause hypoxemia in the first few days of life. CHD with impaired perfusion rather than oxygenation will, though, remain undetected by pulse oximetry. The addition of PPI, a derivative of the quantitative analysis of the pulsatile vs nonpulsatile signal of the pulse oximetry, is a promising technique to cover the diagnostic gaps for this population. 


\section{Disclosure}

The authors report no conflicts of interest in this work.

\section{References}

1. Hoffman JI, Kaplan S. The incidence of congenital heart disease. J Am Coll Cardiol. 2002;39(12):1890-1900.

2. Mahle WT, Newburger JW, Matherne GP, et al; American Heart Association Congenital Heart Defects Committee of the Council on Cardiovascular Disease in the Young; Council on Cardiovascular Nursing; Interdisciplinary Council on Quality of Care and Outcomes Research; American Academy of Pediatrics Section on Cardiology and Cardiac Surgery; Committee on Fetus and Newborn. Role of pulse oximetry in examining newborns for congenital heart disease: a scientific statement from the American Heart Association and American Academy of Pediatrics. Circulation. 2009;120(5):447-458.

3. Schultz AH, Localio AR, Clark BJ, Ravishankar C, Videon N, Kimmel SE. Epidemiologic features of the presentation of critical congenital heart disease: implications for screening. Pediatrics. 2008; 121(4):751-757.

4. $\mathrm{Ng} \mathrm{B}$, Hokanson J. Missed congenital heart disease in neonates. Congenit Heart Dis. 2010;5(3):292-296.

5. Riehle-Colarusso T, Strickland MJ, Reller MD, et al. Improving the quality of surveillance data on congenital heart defects in the metropolitan Atlanta congenital defects program. Birth Defects Res A Clin Mol Teratol. 2007;79(11):743-753.

6. Mahle WT, Martin GR, Beekman RH 3rd, Morrow WR; Section on Cardiology and Cardiac Surgery Executive Committee. Endorsement of Health and Human Services recommendation for pulse oximetry screening for critical congenital heart disease. Pediatrics. 2012;129(1):190-192.

7. Frank LH, Bradshaw E, Beekman R, Mahle WT, Martin GR. Critical congenital heart disease screening using pulse oximetry. $J$ Pediatr. 2013;162(3):445-453.

8. Wren C, Reinhardt Z, Khawaja K. Twenty-year trends in diagnosis of life-threatening neonatal cardiovascular malformations. Arch Dis Child Fetal Neonatal Ed. 2008;93(1):F33-F35.

9. Studer MA, Smith AE, Lustik MB, Carr MR. Newborn pulse oximetry screening to detect critical congenital heart disease. J Pediatr. 2014;164(3):.e1-.e2.

10. de Wahl Granelli A, Mellander M, Sunnegårdh J, Sandberg K, OstmanSmith I. Screening for duct-dependant congenital heart disease with pulse oximetry: a critical evaluation of strategies to maximize sensitivity. Acta Paediatr. 2005;94(11):1590-1596.

11. Kemper AR, Mahle WT, Martin GR, et al. Strategies for implementing screening for critical congenital heart disease. Pediatrics. 2011;128(5):e1259-e1267.

12. Garg LF, Van Naarden Braun K, Knapp MM, et al. Results from the New Jersey statewide critical congenital heart defects screening program. Pediatrics. 2013;132(2):e314-e323.

13. Kochilas LK, Lohr JL, Bruhn E, et al. Implementation of critical congenital heart disease screening in Minnesota. Pediatrics 2013;132(3):e587-e594.

14. Kochilas LK, Menk JS, Saarinen A, Gaviglio A, Lohr JL. A comparison of retesting rates using alternative testing algorithms in the pilot implementation of critical congenital heart disease screening in Minnesota. Pediatr Cardiol. 2015;36(3):550-554.
15. McDermott TL, Vernon MM, Schultz AH. Voluntary implementation of critical congenital heart disease screening in Washington hospitals. Hosp Pediatr. 2015;5(4):193-202.

16. Fouzas S, Priftis KN, Anthracopoulos MB. Pulse oximetry in pediatric practice. Pediatrics. 2011;128(4):740-752.

17. Poets CF, Southall DP. Noninvasive monitoring of oxygenation in infants and children: practical considerations and areas of concern. Pediatrics. 1994;93(5):737-746.

18. Kelleher JF, Ruff RH. The penumbra effect: vasomotion-dependent pulse oximeter artifact due to probe malposition. Anesthesiology. 1989;71(5):787-791.

19. Hanning CD, Alexander-Williams JM. Pulse oximetry: a practical review. BMJ. 1995;311(7001):367-370.

20. Moyle JT. Uses and abuses of pulse oximetry. Arch Dis Child. 1996; 74(1):77-80.

21. Levesque BM, Pollack P, Griffin BE, Nielsen HC. Pulse oximetry: what's normal in the newborn nursery? Pediatr Pulmonol. 2000;30(5): 406-412.

22. de Wahl Granelli A, Wennergren M, Sandberg K, et al. Impact of pulse oximetry screening on the detection of duct dependent congenital heart disease: a Swedish prospective screening study in 39,821 newborns. BMJ. 2009;338:a3037.

23. Ewer AK. Pulse oximetry screening: do we have enough evidence now? Lancet. 2014;384(9945):725-726.

24. Ewer AK, Granelli AD, Manzoni P, Sánchez Luna M, Martin GR. Pulse oximetry screening for congenital heart defects. Lancet. 2013; 382(9895):856-857.

25. Ewer AK, Middleton LJ, Furmston AT, et al; PulseOx Study Group. Pulse oximetry screening for congenital heart defects in newborn infants (PulseOx): a test accuracy study. Lancet. 2011;378(9793):785-794.

26. Reich JD, Connolly B, Bradley G, et al. Reliability of a single pulse oximetry reading as a screening test for congenital heart disease in otherwise asymptomatic newborn infants: the importance of human factors. Pediatr Cardiol. 2008;29(2):371-376

27. Singh A, Ewer AK. Pulse oximetry screening for critical congenital heart defects: a UK national survey. Lancet. 2013;381(9866):535.

28. Thangaratinam S, Brown K, Zamora J, Khan KS, Ewer AK. Pulse oximetry screening for critical congenital heart defects in asymptomatic newborn babies: a systematic review and meta-analysis. Lancet. 2012;379(9835):2459-2464.

29. Hales JR, Stephens FR, Fawcett AA, et al. Observations on a new non-invasive monitor of skin blood flow. Clin Exp Pharmacol Physiol. 1989;16(5):403-415.

30. Lima AP, Beelen P, Bakker J. Use of a peripheral perfusion index derived from the pulse oximetry signal as a noninvasive indicator of perfusion. Crit Care Med. 2002;30(6):1210-1213.

31. Granelli A, Ostman-Smith I. Noninvasive peripheral perfusion index as a possible tool for screening for critical left heart obstruction. Acta Paediatr. 2007;96(10):1455-1459.

32. Peterson C, Grosse SD, Glidewell J, et al. A public health economic assessment of hospitals' cost to screen newborns for critical congenital heart disease. Public Health Rep. 2014;129:86-93.

33. Peterson C, Grosse SD, Oster ME, Olney RS, Cassell CH. Costeffectiveness of routine screening for critical congenital heart disease in US newborns. Pediatrics. 2013;132:e595-e603.
Medical Devices: Evidence and Research

\section{Publish your work in this journal}

Medical Devices: Evidence and Research is an international, peerreviewed, open access journal that focuses on the evidence, technology, research, and expert opinion supporting the use and application of medical devices in the diagnosis, treatment and management of clinical conditions and physiological processes. The identification of novel

\section{Dovepress}

devices and optimal use of existing devices which will lead to improved clinical outcomes and more effective patient management and safety is a key feature. The manuscript management system is completely online and includes a quick and fair peer-review system. Visit http://www. dovepress.com/testimonials.php to read real quotes from authors. 